\title{
Have we forgotten the meaning of the word "doctor"?
}

Volume 3 Issue I - 2016

\section{Editorial}

Doctor is a Latin word, and it was borrowed from Latin already formed, with a meaning, namely 'teacher'. The word is formed exactly the way teacher is:
i. A verb root (English teach-, Latin doc-), plus
ii. An agentive suffix (English -er, Latin -tor).

When I took this vow 25years ago, to step into the "doctor" cloak, I became dedicated to acquiring as much wisdom as possible, in my journey of the standard of care used today $=>$ clinical practice. Being present, as I learned to facilitate healing in others, I was along for the ride doing same. Clinical experience catalyzed the evolution of my learning how to best serve each person seeking guidance, collaborating with each individual who was willing to become a student, their story was revealed with each encounter. As I morphed from student to teacher and back again, in an eternal cycle of living my purpose, seeking to leave a significant legacy that would/could transform the application of the healing arts, with awareness that we are all connected, transmuting energies as we come together to cocreate desired outcomes.

I gladly, joyously, now heed a loud call to teach what I have learned to other practitioners, realizing that my desire is to impart the wisdom I have gathered, empowering future generations whose purpose aligns with mine. By providing these young doctors with effective step-by-step strategies and tools that will amplify results and shorten prognosis. I believe this journey will have a profound and far reaching impact on the health and wellness of humanity at large. Allowing me the opportunity to leave an imprint/legacy, fulfilling a purposeful life.

I believe that the best way to achieve this lofty goal, I am dedicated to, is to publish/share my findings, in as many forms and ways that will result in lasting, meaningful, impactful trends for centuries to come.

In the human body, everything is connected, as a student of this miraculous and intricately engineered expression, my observations continue to confirm there is an intricate network of infinite permutations of these connections, all wired uniquely within each being and each, in turn, with the entire universe in all of its expression.

I have concluded, that in order to have these networks of connection run "healthy programs for optimal human performance", 3 basic conditions must exist:

a. The internal environment that holds all that constitutes a human body, must have optimal operating conditions.

b. Supplemental nutritional protection must be included, due to the inherent exposure of toxic city living.

c. Strategies and tools to resolve persistent negative thought

\author{
Debbie Novick \\ Novick Integrative Medicine, USA
}

Correspondence: Debbie Novick, Novick Integrative Medicine, I7I Saxony RD, Suite 107, Encinitas, CA 92024 USA, Tel 7605191759, Email drdebnovtep@gmail.com

Received: January 24, 2016 | Published: February 03, 2016

patterns, or long standing self injurious cultural messages. What is believed can be achieved. Gradually delivering tools that reinforce self-acceptance and self awareness, so each can become more connected to who they truly are, reprogramming DNA, and manifesting a more congruent expression of the inseparable body/mind.

Question is: What is under our control?

a. Internal conditions. YES

b. City living toxins- Not to exposure, yes to tolerance

c. Mental-emotional state- YES

Meeting requirement " $\mathrm{A}$ " is a matter of food/ beverage choices $\&$ nutritional supplements, each person's journey of discovery and evolving expression of optimal human performance.

Contributing to minimizing "B", the effects of toxic city life, begins with consuming certain nutritional supplement combinations that improve the body's ability to become more chemically-immune tolerant to exposures over time.

Making a commitment to " $\mathrm{C}$ ", becoming more accountable for the thoughts and words we use, which in themselves, are energy like everything else we are made of, and creates this oneness. The more positive, grateful, honoring, accepting we are of ourselves the easier it is to accept others and life experiences.

Each human body has an intricate feedback system that speaks to us from within, if we only take time to listen to the signal/symptoms, which are guideposts toward unraveling the puzzle that is in the way of fueling the "power that made the body can heal it".

With a collaborative mindset, today's functional medicine "doctor" is capable of unraveling these clues and having a profound effect on the state of health care today.

The contributions in this month's journal speak loudly to the many brilliant minds dedicated to delivering powerful information that is worth considering before your next action step. I invite you to gain a broader more empowered perspective.

Enjoy and keep on learning/teaching doctors!

\section{Acknowledgments}

None. 


\section{Conflicts of interest}

Author declares there are no conflicts of interest.

\section{Funding}

None. 